\title{
Self-focusing and filamentation of optical vortex beams: Spatio-temporal analysis
}

\author{
Georgi Maleshkov ${ }^{1}$, Dragomir N. Neshev ${ }^{2}$, Alexander Dreischuh ${ }^{1,2}$ \\ ${ }^{1}$ Department of Quantum Electronics, Faculty of Physics, Sofia University, Sofia-1164, Bulgaria \\ ${ }^{2}$ Nonlinear Physics Centre, Research School of Physical Sciences and Engineering, \\ Australian National University, Canberra ACT 0200, Australia
}

\begin{abstract}
We report numerical simulations supported by experimental observations of self-focusing, fillamentation, and supercontinuum generation by an optical vortex beam in a Kerr nonlinear medium in the regime of dominating nonlinearity. Despite the strong self-focusing resulting in multiple filaments ordered along the vortex ring the optical vortex remains well preserved at the exit of the nonlinear medium and in the far-field. The presented quasi- $(3+1)$ dimensional numerical simulations under azimuthal initial vortex ring perturbations confirm qualitatively the experimentally observed survival of the optical vortex in the course of the white light generation.
\end{abstract}

Keyword list: Nonlinear optics, optical vortex, self-focusing, fillamentation, supercontinuum generation

\section{INTRODUCTION}

The presence of phase dislocations in the wavefront of a light beam determines its phase and intensity structure. Since the phase becomes indeterminate at the singularity point, both the real and the imaginary parts of the field amplitude (i.e., also the field intensity) vanish ${ }^{1}$. An isolated point singularity with a screw-type phase distribution is associated with an optical vortex (OV). The characteristic helical phase profiles of OVs are described by $\exp \{i m \theta\}$-multipliers, where $\theta$ is the azimuthal coordinate and the integer number $m$ is the OV topological charge. The study of OVs has received a special attention in recent years not only because of their rich linear and nonlinear dynamics but also due to a variety of potential applications including particle micro-manipulation ${ }^{2}$ imaging $^{3}$, interferometry ${ }^{4}$, and quantum information ${ }^{5}$.

For applications of OVs in nonlinear optics, ultrashort pulses carrying spatial phase dislocations are an essential element, since such pulses exhibit peak intensities high enough to access optical nonlinearities in many materials. The challenge in creating spatial phase dislocations in short laser pulses with broad spectral bandwidths is to impose the phase dislocation onto all spectral components, while keeping the pulse width and shape undistorted. The known methods for generating phase singularities applicable in the $c w$ and quasi- $c w$ regimes are not suited for femtosecond lasers (see the short overview ${ }^{6}$ ). Well suited for this purpose are optical systems utilizing computer generated hologram or a spatial light modulator aligned within optical schemes with spatial dispersion compensation - dispersionless $4 f^{-6-8}$ or $2 f$-2f-setup ${ }^{9}$, as well as double-pass grating compressor ${ }^{7}$. An achromatic vortex lens comprising pair of suitable adjacent optical glasses whose interface resembles a helicoid seems to be able to create an OV free of topological dispersion ${ }^{10}$ within a bandwidth of $140 \mathrm{~nm}$ in the visible spectral range ${ }^{11}$. Coherent white-light optical vortices generated from supercontinuum Gaussian beam transformed by a spatial light modulator are reported ${ }^{12}$. Polychromatic single- and double-charge OV solitons spanning in bandwidth over more than $70 \mathrm{~nm}$ and $180 \mathrm{~nm}$, respectively, are generated ${ }^{13}$ by focusing a supercontinuum light in a uniaxial potassium titanium oxide phosphate crystal ${ }^{14,15}$ and then propagating the resulting beam in a solid-state nonlinear medium (iron-doped lithium niobate).

In this work we model numerically an alternative approach for generation of white light beams of complex phase structure, in which the OV is first generated in the host femtosecond laser beam, which is subsequently focused in a Kerr nonlinear medium in the regime of dominating nonlinearity. Inspecting the computed near- and far-field energy density and phase distributions we obtain evidences for the remarkable stability of the OV, which survives the selffocusing and the filamentation of the bright vortex ring.

International Conference on Ultrafast and Nonlinear Optics 2009, edited by Solomon Saltiel, Alexander Dreischuh, Ivan Christov, Proc. of SPIE Vol. 7501 $75010 \mathrm{G}$ - @ 2009 SPIE - CCC code: 0277-786X/09/\$18 - doi: 10.1117/12.851352 


\section{THEORETICAL MODEL}

The general procedure we adopt in simulating the OV evolution is to model dominating nonlinear propagation regime inside the nonlinear medium (NLM). For this purpose we solve numerically the $(2+1)$-dimensional nonlinear Schrödinger equation (NLSE)

$$
i \frac{\partial A}{\partial\left(z / L_{D i f f}\right)}+(1 / 2) \Delta_{T} A-\left(L_{D i f f} / L_{N L}\right)|A|^{2} A=0,
$$

which accounts for both the nonlinearity and diffraction, while treating the time as a parameter. This implies that the pulse dispersion length is assumed to be much longer than the nonlinear length. In the NLSE Eq. (1), $\Delta_{T}$ is the transverse part of the Laplace operator whereas $L_{D i f f}$ and $L_{N L}$ stand for the diffraction and nonlinear length of the beam, respectively. In order to account for the time variation of the pulse amplitude/intensity in time we assumed sech-shaped pulse and divided it into $4 N$ time slices, each one having duration $\tau_{S L I C E}=\tau_{H W 1 / e} / N$. The minimum slice number required $(N=50)$ was determined from both the energy conservation and the reproducibility of the transverse intensity distribution in the time-integrated picture against increasing $N$ twice. In order to be able to track the change of each slice from the exit of the NLM to the observation plane located several diffraction lengths away, the computational grid spanned over 1024x1024 points. The local variation (in time) of the pulse amplitude/intensity causes different evolution of the beam perturbation in each time slice. Since the model requires calculations at different local intensities to reflect the intensity decrease in both the pulse leading and trailing wings, the propagation distance is further expressed in units of $L_{\text {Diff }}$, not in nonlinear lengths.

An important first step in the analysis is to calibrate the peak intensity used in the numerical simulations. Let us assume that the one-dimensional laser beam is Gaussian $\left(A=A_{0} \exp \left\{-r^{2} / \omega_{0}^{2}\right\}\right)$ with a half-width at $1 / e$-level equal to $\omega_{0}$. The beam width evolves in vicinity of the beam waist according to the known relation $\omega^{2}(z)=\omega_{0}^{2}(z=0)\left[1+\left(z / L_{D i f f}\right)^{2}\right]$, where $L_{D i f f}=k \omega_{0}^{2} / 2$ is the diffraction length and $k$ is the wave-number. For a onedimensional (1D) bright beam of a width equal to this of the bright vortex ring we estimated that $L_{\text {Diff }}=8$ arb. units and the intensity needed to form an $1 \mathrm{D}$ bright soliton is $I_{S O L}^{1 D}=1.76$.

For describing the OV beam transverse profile we used the self-similar function ${ }^{16}$

$$
A(r, z)=A(r, z=0)\left(\frac{\omega_{0}}{\omega(z)}\right)\left(\frac{r}{\omega(z)}\right)^{|m|} \exp \left\{-\frac{r^{2}}{\omega^{2}(z)}\right\} \exp \{\operatorname{im} \theta\}
$$

representing an $r$-vortex in a Gaussian beam in which $m$ is the vortex topological charge and $\theta$ is the azimuthal coordinate. (For $|m|=0$ it describes the propagation of a Gaussian $\mathrm{TEM}_{00}$ mode.) In order to match the bright vortex ring beam amplitude to this of the 1D bright beam we multiplied the pulse amplitude by a factor of 1.474 . Increasing the field amplitude by a second factor of $\sqrt{2}$ we still observed vortex ring broadening along the NLM, whereas at a factor of 2 a well pronounced bright vortex ring self-focusing is clearly seen at $z=6 L_{D I F F}$.

We analyze the vortex propagation dynamics under two different types of initial perturbations: a $1 \mathrm{D}$ and 2D-azimuthal ones. The amplitude of the analyzed singly-charged OV beam with purely 1D perturbation is described by

$$
\tilde{A}(r, z=0)=\left(r / \omega_{0}\right)[1+\delta \cos (\alpha y)] \exp \left\{-r^{2} / \omega_{0}^{2}\right\} \exp \{i \theta\},
$$

where $\delta$ is the perturbation amplitude and $\alpha$ is the its transverse wave number determining the period of the perturbation. The azimuthal perturbation is described by

$$
\tilde{A}(r, z=0)=\left(r / \omega_{0}\right)[1+\delta \cos (\beta \vartheta)] \exp \left\{-r^{2} / \omega_{0}^{2}\right\} \exp \{i \theta\},
$$

where $\beta$ is an integer number inversely proportional to the azimuthal period of the perturbation. 


\subsection{One-dimensional perturbation}

\section{NUMERICAL RESULTS}

In order to gain systematic insight in the OV evolution in self-focusing Kerr nonlinear medium we first model the intensity dependence of the development of the 1D perturbation at the pulse maximum (local time $t=0$ ) and at a fixed length of the NLM $\left(z / L_{D I F F}=6\right)$. The input OV intensity and phase profiles described analytically by Eq. 2 are shown in Fig. 1. The OV phase profile (Fig. 1, right frame) is particularly important in order to recognize the presence of the vortex and its position when the amplitude/intensity distribution of the beam becomes more complicated.
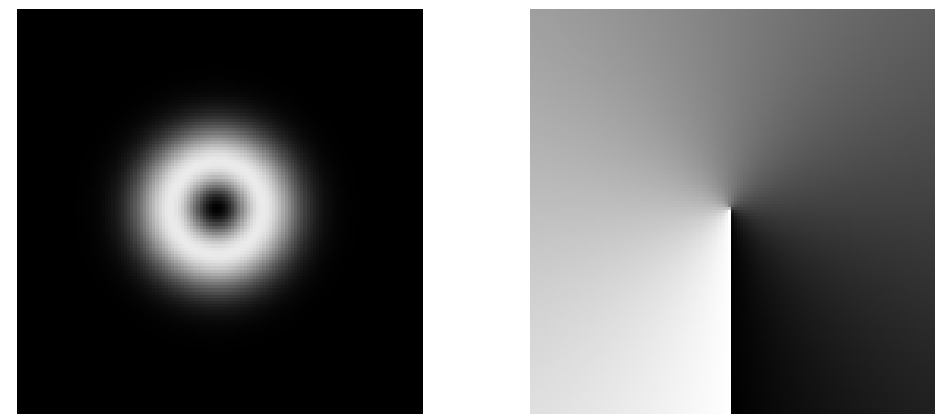

Figure 1. Intensity (left) and phase profile (right) of the unperturbed vortex ring.

The strong 1D modulation at the entrance facet of the NLM $(\delta=0.24, \alpha=5)$ was proven to give qualitatively the same results as these obtained for a $5 \%$ perturbation $(\delta=0.05)$ but at a higher vortex-ring sub-beam dynamics. Generally, the vortex ring decays into pairs of bright filaments which rotate and repel. At a fixed propagation distance inside the NLM both the rotation and repulsion increase with increasing the vortex intensity. Interestingly, after initial shrinking, the two self-focusing sub-beams start diffracting strongly, another pair self-focuses and, at certain distances, the dominating pair of sub-beams changes. This is clearly seen in frames (b) and (c) in Fig. 2.


Figure 2. Sub-beams generated the one-dimensional instability of the vortex ring at (left to right) $I / I_{S O L}^{1 D}=1.75,3.25,3.75$, and 4 for $z / L_{\text {Diff }}=6$. Initial modulation depth $24 \%$. Some $4.7 \%$ of the total computational area is shown.

It should be pointed out that in experiments carried out with (ultrashort) laser pulses the local intensity is the highest in the pulse center (local time $t=0$ ) and decreases in the pulse leading and trailing wings. Considering the time as a parameter (i.e. neglecting the group-velocity dispersion and the eventual pulse reshaping due to e.g. the intensity dependence of the group velocity) the evolution of the beam at different local times should be expected to be different. Therefore, the time-integrated energy density distribution of the OV ring at the exit of the NLM and in the far field should be expected to be gradually different. This is clearly visible in the simulated near-field image shown in Fig. 3, which is calculated for $I(t=0) / I_{S O L}^{1 D}=4$ and sech-shaped pulse divided into 200 time slices with a half-width at $1 / e$ level represented by 50 time slices. The result shown in Fig. 3 is spectacular with the presence of multiple pairs of selffocusing filaments at different filament-to-filament distances and different rotation angles. In a real experiment these filaments should be the sources of supercontinuum. Besides the rather complicated intensity distributions, one can see the vortex ring in the background, which comes from the less intense time wings of the pulse. This result, even slightly 
overdosed in intensity and modulation depth and approaching the border of validity of the slowly-varying envelope approximation, under which Eq. (1) holds, motivated us to look for more moderate conditions, which better fit to the experimental observation (see Sec. 4).

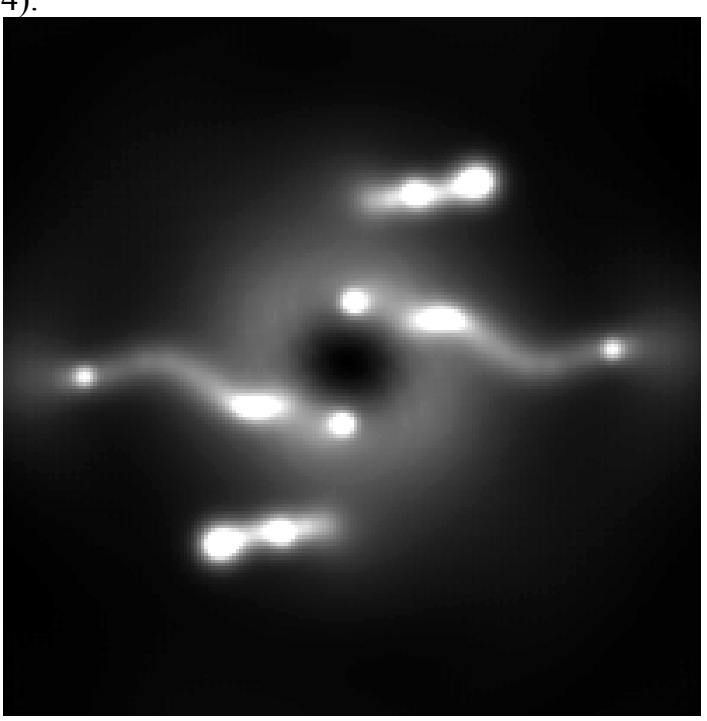

Figure 3. Time-integrated picture showing that the vortex ring in the pulse wings survives the instability. Bright spots - bright filaments born by the modulational instability. $I(t=0) / I_{S O L}^{1 D}=4$ and $z / L_{D i f f}=6$. Some $3.8 \%$ of the total computational area is shown. Initial modulation depth $24 \%$.

All further results presented in this work refer to an initial modulation depth $\delta=0.05$. We restricted the maximum input vortex ring intensity to values, which allow observing filament formation but at filament intensities at the exit of the NLM, which are below 8 times the input vortex ring intensity. In Fig. 4 we show the corresponding simulation results similar to Fig. 2, but for modulation depth $\delta=0.05$. The highest intensity $I(t=0) / I_{S O L}^{1 D}=2.2$ is chosen to correspond to the maximum peak intensity of the vortex ring (i.e. to a local time $t=0$ ).
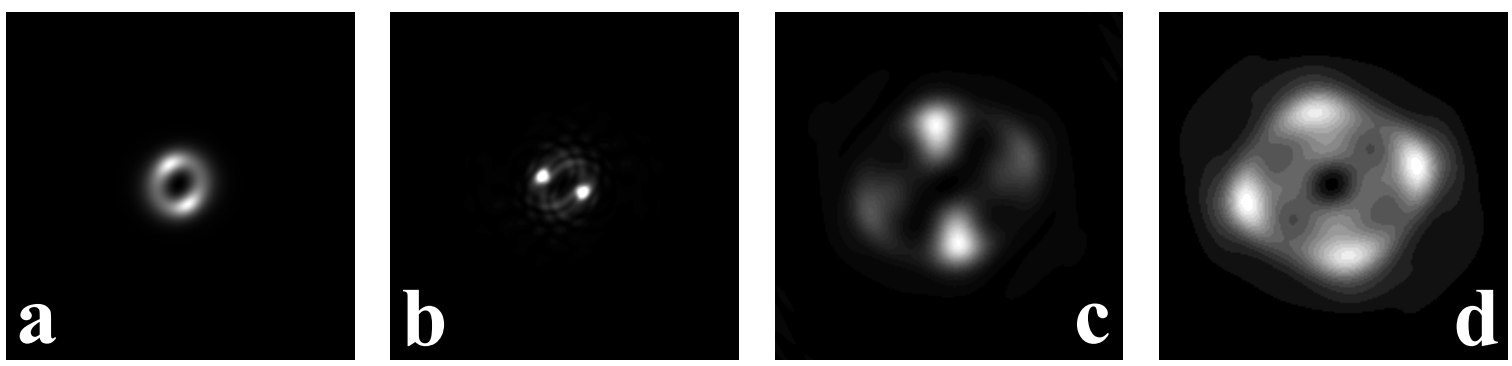

Figure 4. Decay of the vortex ring by one-dimensional perturbation at (left to right) $I(t) / I_{S O L}^{1 D}=1.8,2.0,2.1$, and 2.2 for $z / L_{D i f f}=6$. Initial modulation depth $5 \%$. Some $3.8 \%$ of the total computational area is shown.

Increasing the peak intensity one can clearly see the same tendency - vortex ring breakup into filaments, filament rotation, mutual repulsion, and change in the dominating filament pair. Fig. 5(a) shows the corresponding rotation angle of the filament pair with respect to the y-axis and Fig. 5(b) shows the distance between the filaments. The curves in Fig. 5 are discontinuous because at certain intensity a different filament pair starts dominating. In these simulations we clearly observed that the helical phase profile of the OVs remains well preserved in all time slices, i.e. the OV survives the process of vortex-ring breakup due to the modulational instability in self-focusing Kerr medium. This is well seen in the phase profiles of the waves at the exit of the NLM (Fig. 6b) and after free space propagation at a distance $z=6 L_{D i f f}$ after the NLM (Fig. 6d). The linear propagation distance in our simulations is limited by the size of 
the computational window, however the images fairly represent the far field of the beam. The observe stability of the OV beam is in contrast to earlier studies of vortex break-up ${ }^{17.18}$ and is due to the short propagation length in the NLM.
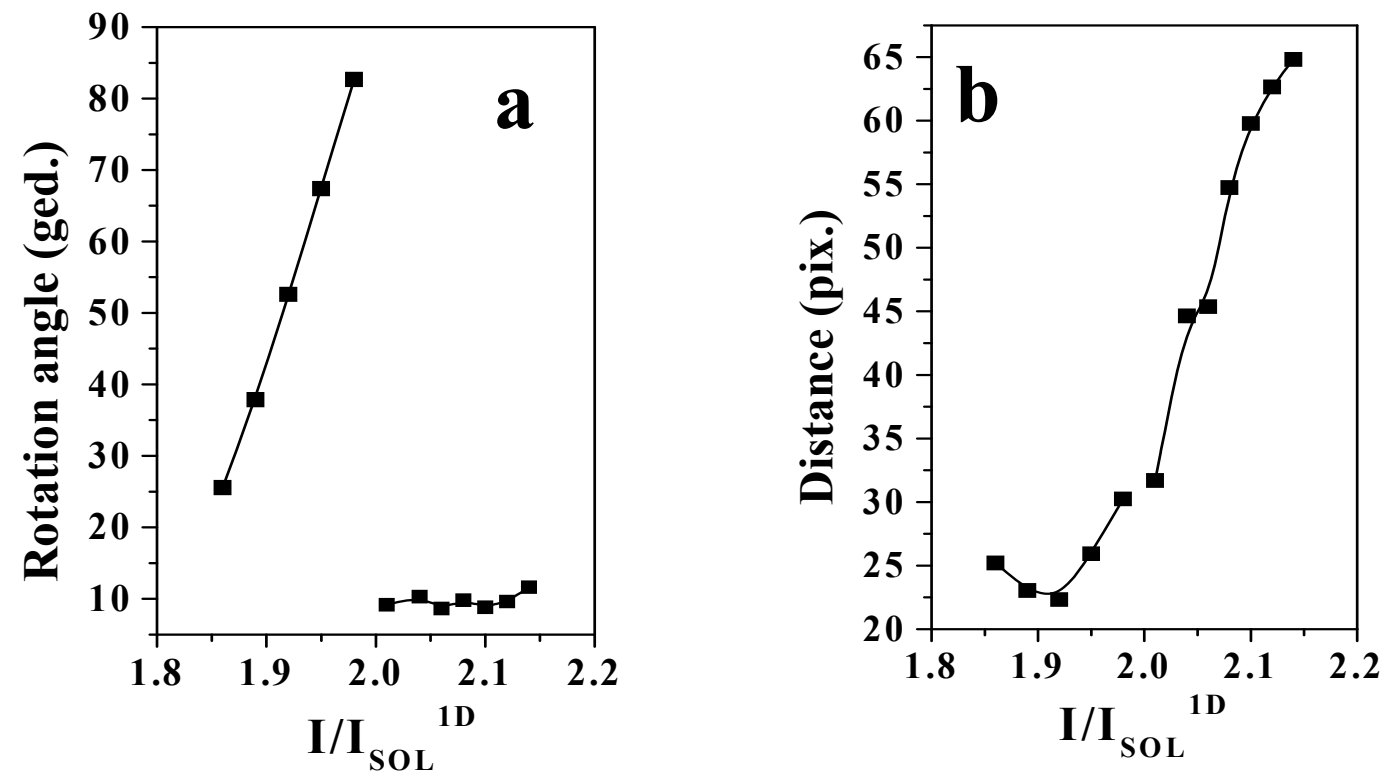

Figure 5. Rotation angle (a) and distance between the decayed two filaments (b) vs. intensity. Initial modulation depth 5\%.
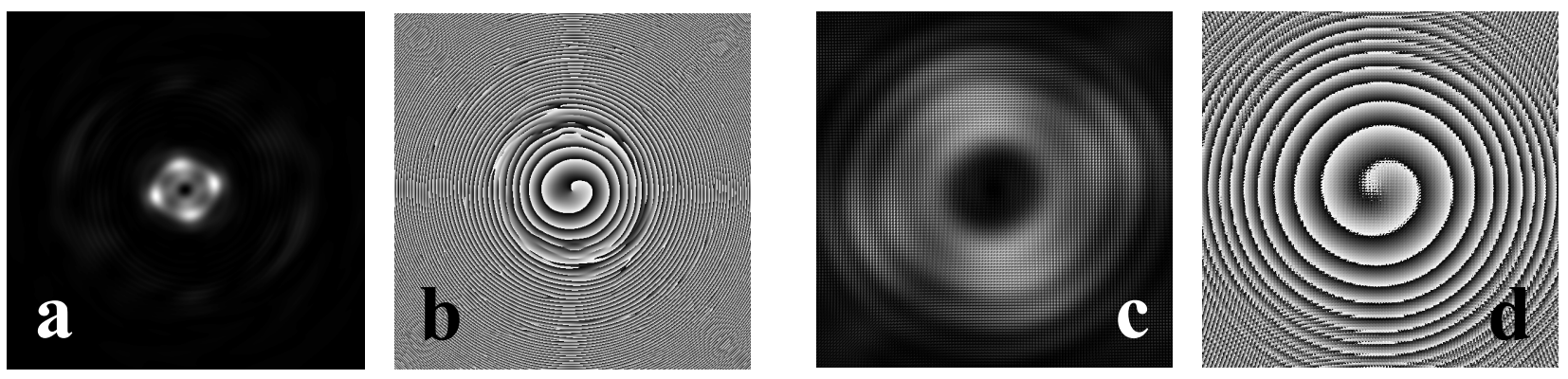

Figure 6. Central slices at local time $t=0\left(I(t=0) / I_{S O L}^{1 D}=2.2\right)$ of the decayed vortex ring at the exit of the NLM (a,b) and after a free propagation distance $z / L_{\text {Diff }}=6$ behind the $\operatorname{NLM}(\mathrm{c}, \mathrm{d})$. Initial modulation depth $5 \%$. Some $34 \%$ of the total computational area is shown. $(\mathrm{a}, \mathrm{c})$ - intensity distributions, $(\mathrm{b}, \mathrm{d})$ - phase profiles.

In the left frame in Fig. 7 we show time-integrated energy density profile of the vortex ring at the exit of the NLM. The two bright spots are self-focusing filaments born from the decayed intense parts of the vortex ring. The halolike structure around the vortex core comes from the less intense parts of the beam/pulse. It is clearly seen even without inspecting the phase profiles of the time slices at different intensities that the OV remains well preserved during the whole pulse at both the exit of the NLM (Fig. 7, left frame) and after a linear propagation over $z=6 L_{\text {Diff }}$ behind the NLM (Fig. 7, right frame). The broad halo around the vortex ring at $z=6 L_{\text {Diff }}$ behind the NLM is caused by the strongly diffracting filaments and, in real experimental conditions, should be expected to be of a very broad spectral bandwidth (white light background). 

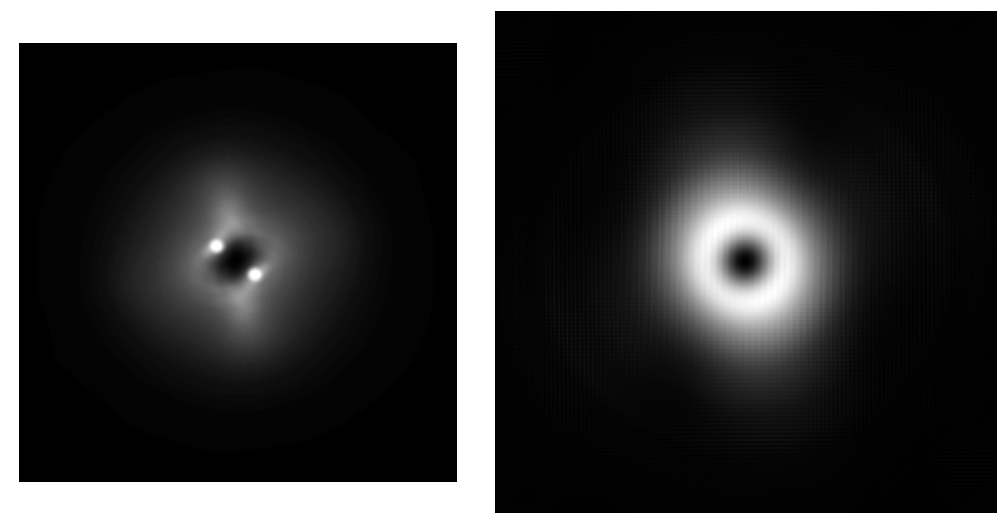

Figure 7. Time-integrated energy density profile of the vortex ring at the exit of the NLM (left) and time-integrated distribution of the OV beam at $z / L_{\text {Diff }}=6$ behind the NLM (right). Initial one-dimensional perturbation of a $5 \%$ depth. $I(t=0) / I_{S O L}^{1 D}=2.2$. Note that in the left frame some $3.8 \%$ of the total computational area is shown, whereas in the right frame $34 \%$ of this area is shown.

\subsection{Two-dimensional (azimuthal) perturbation}

Next, we systematically modeled the evolution of a vortex ring with an initial azimuthal perturbation (Eq. (4)) of a modulation depth of 5\%. For four azimuthal modulation periods and the same nonlinear propagation distance we observed symmetric decay of the vortex ring into four sub-beams, which clearly self-focus (at sufficiently high intensity $\left.I(t) / I_{S O L}^{1 D}=2.2-4\right)$, repel each other and rotate as an ordered structure. These results are qualitatively similar to the results obtained with gradually broader vortex rings discussed below.

To better match the experimental observations (see Section 4) we consider the evolution of the vortex ring in the case of a 2D azimuthal perturbation of 12 periods (see the upper left frame in Fig. 8) when the input vortex ring has 4 times higher width as compared to the vortex ring modeled previously. The peak intensity $\left.I(t=0) / I_{S O L}^{1 D}=0.8\right)$ and the nonlinear propagation length $\left(\mathrm{z} / \mathrm{L}_{\text {Diff }}=0.375\right)$ are scaled accordingly. At high intensity the axially-symmetric perturbation forces the vortex ring to decay into axially-symmetric set of filaments (see the frame at $t=0$ ). The decrease of the local intensity (at increased local times) decreases the growth-rate of the modulation instability and the vortex ring experiences weaker and weaker self-focuses, approaching the regime of linear diffraction far in the leading and trailing wings of the pulse. This tendency is obvious from the data shown in Fig. 8.
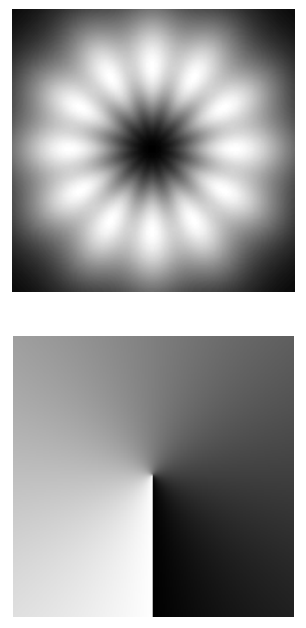

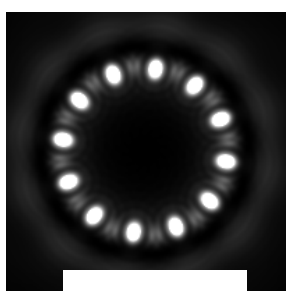

$$
\boldsymbol{t}=\mathbf{0}
$$

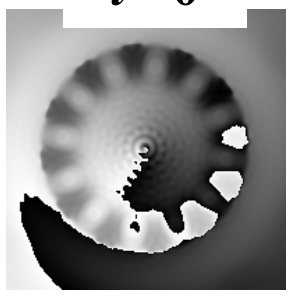

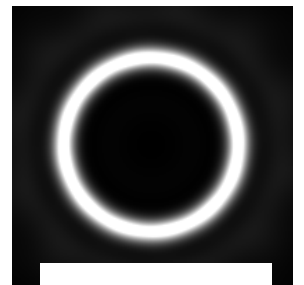



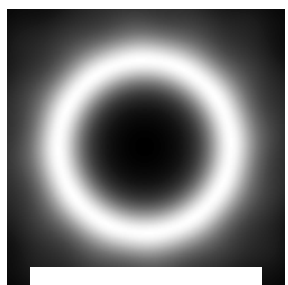

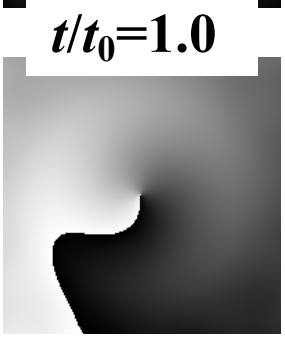

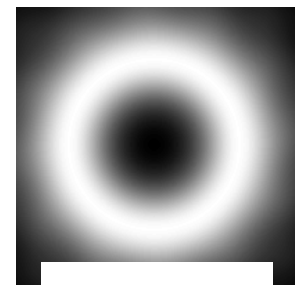

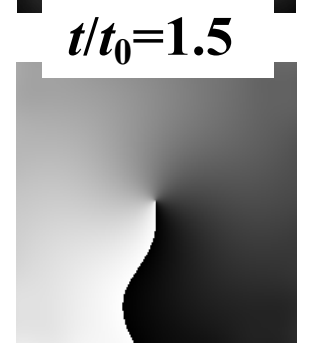

Figure 8. Intensity (upper row) and phase profiles (lower row) of the initial azimuthally perturbed vortex ring (left column) and respective energy density profiles at the exit of the NLM at local times (left to right) $t / t_{0}=0,0.5,1.0$, and 1.5 . Note that the phase profiles confirm the presence of an optical vortex in each time slice and, hence, in the time-integrated picture. $5 \%$ initial modulation depth. $I(t=0) / I_{S O L}^{1 D}=0.8$ and $\mathrm{z} / \mathrm{L}_{\mathrm{Diff}}=0.375$. Some $3.8 \%$ of the total computational area is shown in each frame. 
It is important to underline here that the well preserved helical phase profiles at each local time confirm the presence of an optical vortex in each time slice and, hence, in the time-integrated picture. The pictures shown in Fig. 9 clearly confirm this both at the exit of the NLM (left) and after certain linear propagation distance behind the NLM (middle and right images). Under the influence of the diffraction, the OV becomes fully recovered after relatively short free-space propagation distance. The right frame in Fig. 9 is the same as the middle one, but intentionally saturated to show the "background" caused by the diffracting self-focused filaments.
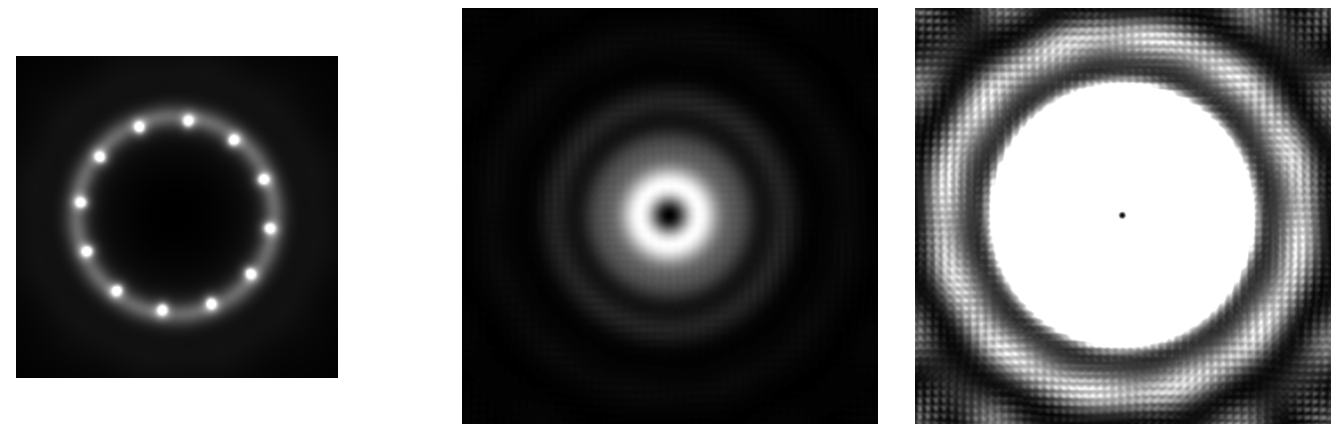

Figure 9. Time-integrated pictures of the vortex ring in the case of a $2 \mathrm{D}$ azimuthal perturbation of 12 periods for $I(t=0) / I_{S O L}^{1 D}=0.8$ at the exit of the NLM (left frame; $z / L_{\text {Diff }}=0.375$ ) and at a distance $z / L_{\text {Diff }}=1.125$ behind the NLM (middle and right frame). Some $3.8 \%$ of the total computational area is shown in the left frame, $34 \%$ in the other two frames. Right frame - the same as in the middle, but intentionally saturated to see the halo around the vortex ring.

Despite the somewhat more complicated evolution of the vortex ring at intermediate stages inside the NLM, we obtained qualitatively the same results in the case of four azimuthal modulation periods when all other model parameters are kept unchanged. The only significant difference is that the vortex ring breakup starts already at $t / t_{0}=1.5$, at $t / t_{0}=0.5$ (i.e. at a higher local intensity) four self-focusing filaments are clearly pronounced, and at the pulse center $(t=0)$ each of them splits further into four sub-peaks. This is visible also in the time-integrated picture at the exit of the NLM (see Fig. 10 , left frame), but in this picture only three sub-filaments are dominating. The diffraction of the structured vortex ring fully recovers the OV ring after relatively short free-space propagation distance $\left(z / L_{\text {Diff }}=1.125\right)$. As done in generating Fig. 9, the right frame in Fig. 10 is the same as the middle one, but saturated to show the "background" caused by the self-focused filaments creating supercontinuum background.
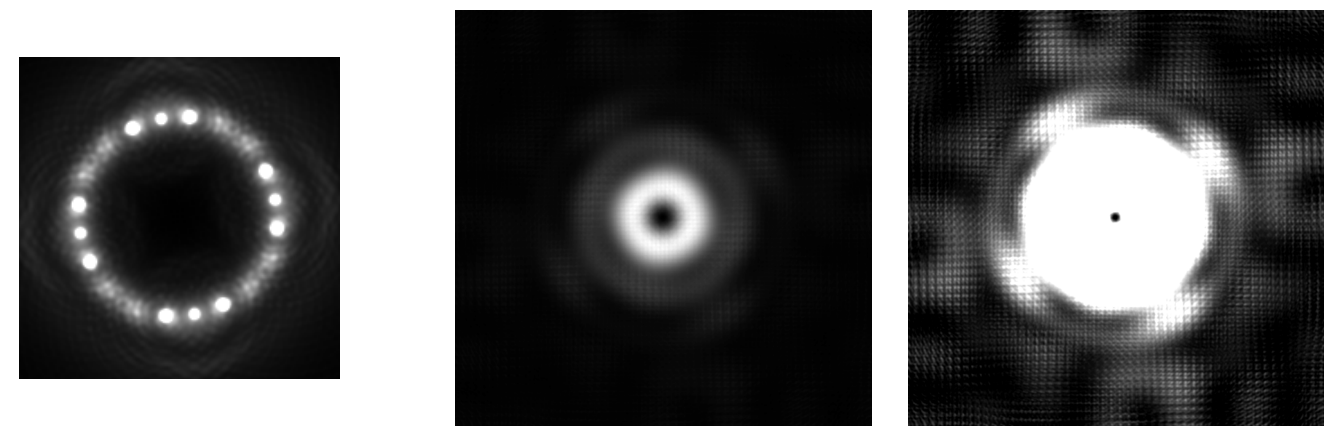

Figure 10. The same as in Fig. 9 but for four azimuthal modulation periods $(\beta=4)$.

\section{KEY EXPERIMENTAL DATA}

In order to confirm the presented numerical simulations, we performed proof-of-principle experiments where we first generated an intense optical vortex beam/pulse and then created and studied the supercontinuum with a screw phase dislocation nested in. Because of the 30\% efficiency of the available computer-generated hologram (CGH) we used a low repetition rate $(250 \mathrm{~Hz})$ chirped-pulse amplification system (CPA-2001, Clark-MXR Inc.) delivering 150-fs pulses of a peak power of $15 \mathrm{MW}$. The $\mathrm{CGH}$ was fabricated by etching of the corresponding vortex interference pattern onto a glass substrate with a grating period of $80 \mu \mathrm{m}$. Because the continuum generation in condensed media is triggered by self-focusing and strongly depends in width on the bandgap $E_{\text {gap }}$ of the material above certain threshold, our choice was 
to use $5 \mathrm{~mm}$ thick sample of $\mathrm{CaF}_{2}\left(E_{\mathrm{gap}}=10.2 \mathrm{eV}\right)$. The sample was rotated in order to prevent possible optical damage. The beam intensity was varied by adjusting the beam waist position with respect to the sample, while keeping the sample-to-imaging lens distance unchanged. Loose focusing done by lenses $L_{1}$ of focal lengths $f=30 \mathrm{~cm}$ or $50 \mathrm{~cm}$ was sufficient to observe well developed supercontinuum generation. The near and far-field images of the beam could be simultaneously recorded on colour CCD cameras.

The most important result from the experiment can be briefly summarized as follows. In agreement with the numerical simulations (see the middle and right frames in Figs. 9 and 10) in the far field we always observed a well preserved optical vortex beam in the background of the generated supercontinuum. The origin of the supercontinuum was clarified by inspecting the transverse near field intensity distribution of the beam at the exit of the sample. In Fig. 11 we show a comparison between low power (upper row) and high power ( $22 \mathrm{~mW}$ average power) optical vortices (bottom row) in the background of the supercontinuum. In the linear images (upper row) it can be seen how the OV becomes focused with decreasing the focusing lens-to-sample distance. The self-focusing in the nonlinear case (lower row) is well pronounced and is accompanied by an increase (from (a) to (c)) of the number of filaments. These filaments generate the supercontinuum radiation which, because of its much stronger diffraction after the exit of the nonlinear medium, appears in the far field as a background for the vortex. As motivated by the intuition and by the numerical simulations, the hot spots appear arranged along the vortex ring, i.e. at positions of high local peak intensity. Despite some deformation, the symmetry of the SC OV remains well pronounced. Note that despite the hot spots, in all frames the OV core remains well preserved.

\section{CONCLUSION}

The reported numerical and experimental results on the generation of femtosecond supercontinuum in a solid state medium by an optical vortex beam show the existence of well preserved optical vortex beam in the background of the generated supercontinuum. In the most intense parts of the (femtosecond) pulse, the OV ring undergoes self-focusing and multiple filamentation resulting in supercontinuum generation. The strong diffraction of the supercontinuum from the filaments after the exit of the NLM gives a white light background on which the OV from the less intense parts of the pulse wings is residing. The numerical simulation carried out on the base of the $(2+1)$-dimensional NLSE treating the time as a parameter confirm qualitatively this scenario of the optical vortex fillamentation, leading to supercontinuum generation.

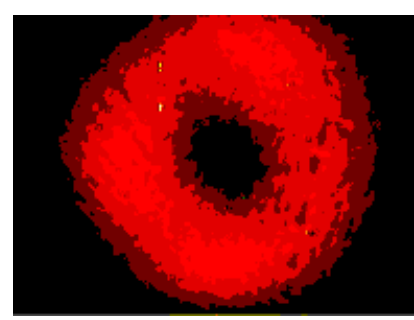

a)

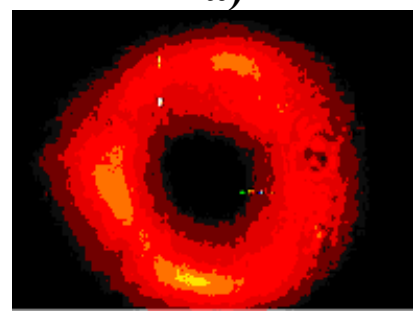

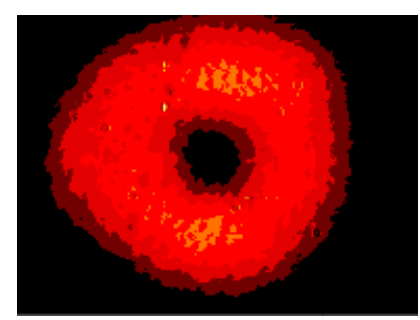

b)

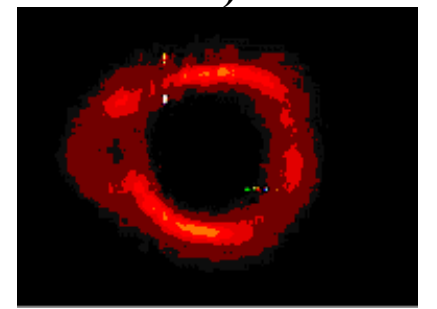

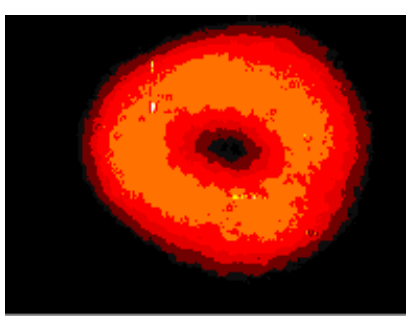

c)

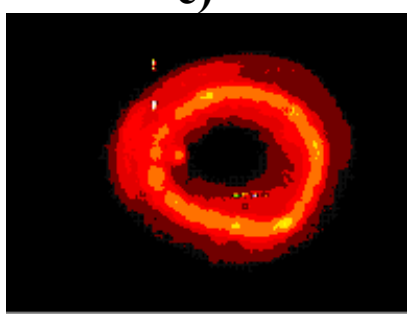

Figure 11. Near-field OV rings observed for low power (upper row) and vortex ring self-focusing and hot spots formation for high power (bottom row; $22 \mathrm{~mW}$ mean power) at decreasing focusing lens-to-sample distance (from a) to c)).

\section{ACKNOWLEDGMENTS}

This work was supported by the National Science Foundation (NSF)-Bulgaria, project WUF-02/2005 and IRNI-17/2007 and by the Australian Research Council through Discovery and Linkage International projects. We acknowledge the useful discussion with Yu. Kivshar and W. Krolikowski, as well as the help with the experiments of M. Samoc. 


\section{REFERENCES}

[1] Nye, J. F. and Berry, M. V., "Dislocations in wave trains," Proc. R. Soc. London A 336, 165-190 (1974).

[2] Grier, D. G., "A revolution in optical manipulation," Nature 424, 810-816 (2003).

[3] Foo, G., Palacios, D. M. and Swartzlander, Jr., G. A., "Optical vortex coronagraph,” Opt. Lett. 30, 3308-3310 (2005).

[4] Furhapter, S., Jesacher, A., Bernet, S. and Ritsch Marte, M., "Spiral interferometry," Opt. Lett. 30, 1953-1955 (2005).

[5] Molina Terriza, G., Torres, J. P. and Torner, L., "Twisted photons," Nature Physics 3, 305-310 (2007).

[6] Besuchanov, K., Dreischuh, A., Paulus, G. G., Schätzel, M. G., Walther, H., Neshev, D., Krolikowski, W. and Kivshar, Yu., "Spatial phase dislocations in femtosecond laser pulses," J. Opt. Soc. Am. B 23, 26-35 (2006).

[7] Besuchanov, K., Dreischuh, A., Schätzel, M. G., Paulus, G. G. and Walther, H., "Vortices in femtosecond laser fields," Opt. Lett. 29, 1942-1944 (2004).

[8] Zeylikovich, I., Sztul, H. I., Kartazaev, V., Le, T. and Alfano, R. R., "Ultrashort Laguerre-Gaussian pulses with angular and group velocity dispersion compensation," Opt. Lett. 32, 2025-2027 (2007).

[9] Mariyenko, I. G., Strohaber, J. and Uiterwaal, C. J. G. J., "Creation of optical vortices in femtosecond pulses," Optics Express 13, 7599-7608 (2005).

[10] Swartzlander, Jr., G. A. and Schmit, J., "Temporal correlation vortices and topological dispersion,” Phys. Rev. Lett. 93, 093901 (2004).

[11] Swartzlander, Jr., G. A., “Achromatic optical vortex lens,” Opt. Lett. 31, 2042-2044 (2006).

[12] Sztul, H. I., Kartazayev, V. and Alfano, R. R., "Laguerre-Gaussian supercontinuum,” Opt. Lett. 31, 2725-2727 (2006).

[13] Neshev, D. N., Dreischuh, A., Shvedov, V., Desyatnikov, A. S., Krolikowski, W. and Kivshar, Yu. S., "Observation of polychromatic vortex solitons," Opt. Lett. 33, 1851-1853 (2008).

[14] Volyar, A., Shvedov, V., Fadeyeva, T., Desyatnikov, A. S., Neshev, D. N., Krolikowski, W. and Kivshar, Yu. S., "Generation of single-charge optical vortices with an uniaxial crystal," Optics Express 14, 3724-3729 (2006).

[15] Shvedov, V., Krolikowski, W., Volyar, A., Neshev, D. N., Desyatnikov, A. S. and Kivshar, Yu. S., "Focusing and correlation properties of white-light optical vortices," Optics Express 13, $7393-7398$ (2005).

[16] Rozas, D., Law, C. T., Swartzlander, Jr., G. A., "Propagation Dynamics of Optical Vortices,” J. Opt. Soc. Am. B 14, 3054-3065 (1997).

[17] Firth, W. J.and Skryabin, D. V., “Optical Solitons Carrying Orbital Angular Momentum,” Phys. Rev. Lett. 79, 2450-2453 (1997).

[18] Vuong, L. T., Grow, T. D., Ishaaya, A., Gaeta, A. L., 't Hooft, G. W., Eliel, E. R. and Fibich, G., "Collapse of optical vortices," Phys. Rev. Lett. 96, 13390(1-4) (2006). 